\title{
A Morphing Technique Applied to Lung Motions in Radiotherapy: Preliminary Results
}

\author{
R. Laurent, J. Henriet, R. Gschwind, L. Makovicka
}

\begin{abstract}
Organ motion leads to dosimetric uncertainties during a patient's treatment. Much work has been done to quantify the dosimetric effects of lung movement during radiation treatment. There is a particular need for a good description and prediction of organ motion. To describe lung motion more precisely, we have examined the possibility of using a computer technique: a morphing algorithm. Morphing is an iterative method which consists of blending one image into another image. To evaluate the use of morphing, Four Dimensions Computed Tomography (4DCT) acquisition of a patient was performed. The lungs were automatically segmented for different phases, and morphing was performed using the end-inspiration and the end-expiration phase scans only. Intermediate morphing files were compared with 4DCT intermediate images. The results showed good agreement between morphing images and 4DCT images: fewer than $2 \%$ of the 512 by 256 voxels were wrongly classified as belonging/not belonging to a lung section. This paper presents preliminary results, and our morphing algorithm needs improvement. We can infer that morphing offers considerable advantages in terms of radiation protection of the patient during the diagnosis phase, handling of artifacts, definition of organ contours and description of organ motion.
\end{abstract}

Keywords: organ motion, morphing, 4DCT.

\section{Introduction}

Organ motion leads to dosimetric uncertainties during a patient's treatment. Much work has been done to quantify the dosimetric effects of lung movement during radiation treatment. In particular, important work is being done on describing and predicting organ motion. Four Dimensions Computed Tomography (4DCT) can easily be performed using Varian's Real-time Position Management System (RPM) with slices acquired throughout the respiratory cycle. A reflective marker is fixed on a box positioned on the chest of the patient. The process is rather complicated, because deformation does not follow the same speed and amplitude at every point of a given organ. Furthermore, in the lungs, hysteresis must be considered: the motion in expiration and inspiration is not symmetrical $[44,45]$. We did not account for hysteresis in this preliminary study.

We had to find a mathematical definition to describe the movement. We chose to use the morphing technique, because it permitted both evaluation and interpolation of movement. Morphing is a computer science which consists of blending one image into another. Turning an image at the beginning of inspiration into an image at the end of the inspiration may therefore provide a precise description of lung motion. Intermediate files obtained with morphing were very close to the $4 \mathrm{DCT}$ intermediate images. One of the most important advantages of morphing over $4 \mathrm{DCT}$ is the fact that the organ position can be precisely known with less exposure to radiation. For this preliminary study, we chose to study the motions of several lungs. We also wish to stress that these results do not yet account for all aspects of organ motion. Nevertheless, the results are promising and encourage further complex studies. Our morphing program has to be further refined before it can be offered for routine use in radiation treatment.

The first section of this paper begins with a brief review of organ motion in radiotherapy, followed by an overview of the simulation of lung motion, as well as an overview of morphing algorithms and tools used in medicine. The second section of the paper presents the material and method used for image acquisition, and also the morphing algorithm. Finally, the results are presented and discussed in the third section.

\subsection{Organ Motions in radiotherapy}

Conformal radiotherapy shapes the treatment fields to conform to the target geometry in three dimensions. However, the use of a single 3DCT (Three Dimensions Computed Tomography) data set for treatment planning assumes that the single CT represents the mean positions of the target organ and of any Organs At Risk (OAR). In reality, the received absorbed dose may differ from the planned absorbed dose. Either the dose coverage of the targeted volume is insufficient or there is an over-dosage of the 
surrounding normal tissue. The motion of the tumour volume is traditionally accounted for by the use of margins according to International Commission on Radiation Units and Measurements (ICRU) reports 50 and $62[14,15]$. The margin used for treatment between the Gross Tumour Volume (GTV) and the Planning Target Volume (PTV) is divided into two parts:

- Internal Margin (IM) to account for Clinical Target Volume (CTV) size and shape variations,

- Setup Margin (SM) to account for uncertainties in patient positioning and beam alignment. The $\mathrm{SM}$ is determined either empirically or by measuring the magnitude of organ motion during respiration.

Many organ motion studies are available in the literature. Generally, they relate to the thoracic or abdominal organs [15], and have used various motion observation techniques: an ultrasound scanner [11], digital fluoroscopy [33], a gamma scintillation camera [43], or a magnetic method [1]. The purposes of these observations are multiple: to improve image acquisition [11, 43], to investigate field margins [33], or to examine effects on organs at risk [1]. Not all imaging modalities are appropriate to all tumour sites. Sixel et al. [33] recommend CT scanner use.

Low et al. [18] studied the motion of a pulmonary tumour with scanner modality and observed that between two breathing extreme ranges one part of the tumour followed a rotational displacement, and the other part followed a stretching displacement. They concluded that a simple one-dimensional rigidmotion model of this tumour would be unable to accurately reflect its motion during the patient's respiratory cycle. Other authors $[4,19]$ have noted that tumours move in translation and simultaneously distort their shape.

Many models and studies exist concerning organ motion prediction. For example, Schweikard et al. [32] studied organ deformation from a mechanical point of view (tissue elasticity) and created a mathematical model, and Heath et al. [13] considered the deformation of a voxel. We decided to explore the possibility of using morphing to simulate organ motion.

\subsection{Simulation of lung motions}

The aim of simulating lung motion is to minimize radiation exposure due to $4 \mathrm{DCT}$ and to help trace the accurate motion of the lung. This knowledge of motion may allow reduced margins to be used in Treatment Planning Systems (TPS) in radiotherapy.

Many works exist on the simulation of lung motions, each with its own method. Villard et al. [41, 42] developed a method based on the Mechanics of Con- tinuous Media laws with resolution by finite elements. This method uses patient data and biomechanical parameters to generate a customized simulation of lung motion.

Boldea et al. [5, 6] estimated lung deformation with an algorithm of non-rigid registration. The implemented algorithm blends one image into another without dimension restriction: the objects represented in the two images may not be represented according to the same dimensions. The two techniques were compared and data similar to a $4 \mathrm{DCT}$ was created. This method was used for all respiratory motions [48].

Santhanam et al. [30] simulated the motions of a surface lung with a tumour to have a model working in real time. The model takes as its input a subject-specific $4 \mathrm{DCT}$ of lungs and computes a deformable lung surface model by estimating the deformation properties of the surface model using an inverse dynamics approach.

However precise the above techniques may be, they are too cumbersome for clinical application as they are based on complicated computations.

\subsection{Morphing for medicine}

This section summarizes the applications of morphing to medicine.

The purpose of morphing is to blend one image into another. Both of the images represent the same object and use the same structure [29].

Morphing presents many potential uses in medicine. J. Talairach and P. Tournoux [36] presented a way to use morphing in order to create a universal cerebral atlas. Others, e.g. J. R. Moringlane [22], designed some morphing algorithms to locate precise points in the brain. Other work has compared and merged data from different patients or has drawn up statistics of distortions. P. M. Thompson and A. W. Toga used morphing to study Alzheimer's disease [37, 39]. E. Stindel used morphing to simulate the evolution of trauma to the patella [34]. B. P. Bergeron et al. [3] developed applications for education based on morphing that are able to generate variations in medical images. K. Penska et al. [25] also used morphing for education and diagnosis by turning radiographic images into a movie that demonstrates the healing process of a humerus fracture. Other examples and approaches were reported by J. Montagner et al. [21].

As presented in Fig. 1, J. B. A. Maintz and M. A. Viergever distinguished four main families of transformations [20]:

1. rigid transformations are simple translations and rotations;

2. affine transformations retain parallelism of image lines; 


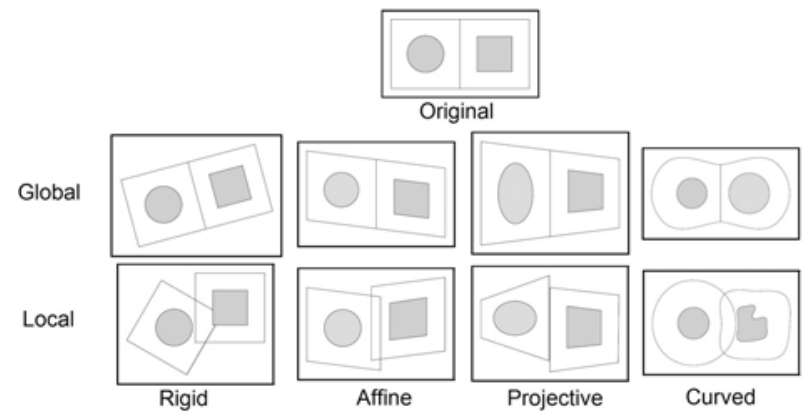

Fig. 1: The four families of transformations through morphing

3. projection transformations retain image lines but not necessarily their parallelism;

4. non-linear transformations turn lines into curves.

Non-linear transformations are usually applied for medical issues. There are three categories of methods for this kind of transformation [23]:

(i) intensity-based methods are based on a global approach over the different grey colour levels. These methods are very complex. Intensitybased methods propose a more global approach. They are based on minimisation of energy, which describes the degree of similarity between the source and the target morphing images. J.-P. Thirion [38] presented a kind of regulation using iterative screening. During this regulation, he drew the outlines of the object in a first image, and then he let the second image filter through the first one. M. Bro-Nielsen and G. Grambow adopted the same approach [7] adding a linear elasticity parameter. C. Davatzikos [10] went further, using the elastic properties of a material. G. E. Christensen added speediness (fluid model) to elasticity [8] and defined constraints using parametric equations. Other multiscale approaches were presented by H. Lester and S. R. Arridge [17]. These approaches implemented either multiple decomposition of the images or the distortions. These transformations are fulfilled according different parameters. The former consisted in solving the transformation of one version of the image into another version and repeating it until the end. The latter, unusually, consists in considering successive distortions with a higher and higher degree of freedom.

(ii) Feature-based methods propose less complex algorithms and faster calculations. Those methods are based on geometrical transformations [47]. Various geometrical methods have been implemented, such as automatic and semi-automatic search and manual search of critical points [26, 40]. The search of crest lines, curve points and shape curves are also geometrical methods that have been implemented [35]. Methods based on the use of models which describe areas are also geometrical methods. These models allow one to roughly extract the most important anatomical structures, and then to refine them [28]. A. L. Didier et al. [12] and D. Sarrut et al. [31] simulated lung motion using geometrical transformations based on continuous mechanical laws, which were solved by the finite element method. This approach takes into account the influence of the organs around the lungs that are responsible for their motion and for limiting their motion: the ribs, the diaphragm, all their associated muscles and the pleura. H. Atoui et al. [2] also implemented a feature-based algorithm to construct missing intermediate lung slices;

(iii) Hybrid methods have also been implemented by L. Collins and A. C. Evans [9].

\section{$2 \quad$ Material and method}

This section presents the materials and methods that are used. In the first part, we described the way the patient data was acquired, and in the second part, we discuss the morphing algorithm that has been implemented.

\subsection{Acquisition of patient data}

With the respiratory gating radiotherapy technique, various tools were developed to register the patient's breathing pattern. Many techniques exist, only two of which are used by the hospital team with which we collaborate: the Real-time Position Management system, (RPM, Varian medical systems, Palo Alto, CA) and the spirometer system. RPM consists of placing a block with two reflective markers on the patient's abdomen in front of a video camera which uses an infrared signal to register the motion of this block. This technique is used in 4D-PET (4 DimensionsPositron Emission Tomography) and 4DCT-scan acquisitions [24, 27], often in free breathing mode. The other technique is the spirometer system, which is often used in breath-hold techniques. The patient's air flow circulates in a spirometer through the mouth [48]. A nose-clip prevents the patient from nasal breathing.

A patient was set up on the 4DCT (GE Lightspeed 16-slice scanner, GE Medical systems, Waukesha, WI) lying in the treatment position, suspended in an alpha cradle, his arms folded above his head. The patient's data was registered in the free breathing cine-mode: the scans were acquired at each table location to ensure a complete sampling of data for one respiratory cycle. The scanning technique used 
$120 \mathrm{kVp}$ and $400 \mathrm{~mA}$ with slices $2.5 \mathrm{~mm}$ in thickness. This process was repeated until the entire thorax was scanned (160 couch positions). The RPM was used to obtain the retrospective 4DCT. These 4DCT images were sorted according to the respiratory pattern. Each 3DCT-scan represents 3D anatomic information at a certain phase. In this study, 4DCT-scans were sorted into ten equal periods. As represented in Fig. 2, $0 \%$ phase corresponds to the end of inspiration and $50 \%$ phase to the end of expiration.

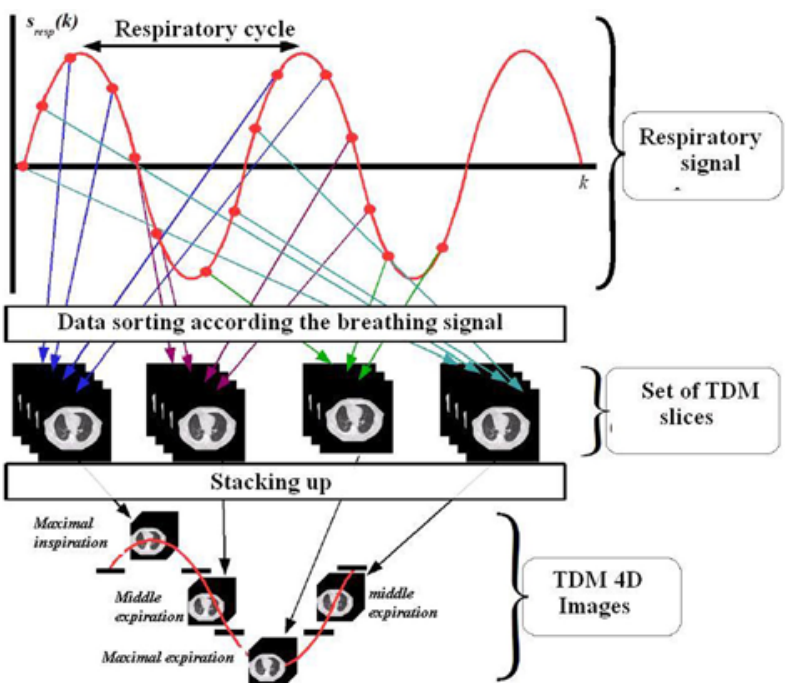

Fig. 2: Respiratory cycle and selection process achieved by $4 \mathrm{DCT}$

Fig. 2 describes the principles of an acquisition performed during one patient's breathing cycle [33]. The respiratory signal and the data are acquired at the same time in order to be correctly sorted. Each 3 D-reconstruction corresponds to one of the six different phases of the respiratory cycle. The entire motion is constituted from the set of $3 \mathrm{D}$ sorted data.

The obtained slices are numbered relatively: Slice 0 is the sagittal slice in the middle of the patient's lung, positive slices are toward the lung apex, and the negatives are toward the diaphragm. It is impossible to obtain one scan that represents each slice at exactly $0 \%, 10 \%, 20 \%, 30 \%, 40 \%$ and $50 \%$ expiration. Consequently a tolerance value is introduced in order to use one scan for each phase. So, the accurate moment does not always correspond to the phase in which it is used. The Advantage4D software (by GE Medical systems) is in charge of this retroclassification. Table 1 shows the correspondences between the accurate moments and the phase in which they are sorted by Advantage4D. As observed in Table 1 , for slices $75,0,-50$ and -100 , the same CT scans are used in the $0 \%$ and $10 \%$ phases leading to artifacts in the final $3 \mathrm{D}$ scan reconstruction. For example, Table 1 shows that slice number 75 was obtained at the precisely $14 \%$ expiration and it is used to construct two 3D images: the image of the $0 \%$ expiration phases and also the image of the $10 \%$ expiration phase.

Table 1: Accurate respiratory moments used for $0 \%$, $10 \%, 20 \%, 30 \%, 40 \%$ and $50 \%$ phases

\begin{tabular}{|c|c|c|c|c|c|c|}
\hline $\begin{array}{c}\text { Slice } \\
\text { Numbers }\end{array}$ & $\begin{array}{c}0 \% \\
\text { phase }\end{array}$ & $\begin{array}{c}10 \% \\
\text { phase }\end{array}$ & $\begin{array}{c}20 \% \\
\text { phase }\end{array}$ & $\begin{array}{c}30 \% \\
\text { phase }\end{array}$ & $\begin{array}{c}40 \% \\
\text { phase }\end{array}$ & $\begin{array}{c}50 \% \\
\text { phase }\end{array}$ \\
\hline 75 & $14 \%$ & $14 \%$ & $22 \%$ & $30 \%$ & $38 \%$ & $53 \%$ \\
\hline 0 & $16 \%$ & $16 \%$ & $16 \%$ & $33 \%$ & $41 \%$ & $50 \%$ \\
\hline-50 & $17 \%$ & $17 \%$ & $17 \%$ & $33 \%$ & $41 \%$ & $49 \%$ \\
\hline-100 & $2 \%$ & $2 \%$ & $34 \%$ & $34 \%$ & $43 \%$ & $52 \%$ \\
\hline
\end{tabular}

Morphing was performed for several couch positions on the first 2D image (classified in $0 \%$ phase) and the final image (classified in $50 \%$ phase). The morphing intermediate files obtained were compared to the intermediate images obtained with 4DCT. All the compared images and intermediate files have the same number of voxels (512 by 256 ). The voxels of all those files have the same dimensions $(28 \mathrm{~mm}$ by $38 \mathrm{~mm}$ by $50 \mathrm{~mm}$ ) and Cartesian coordinates. Thus, to compare one file with another, we counted the number of voxels which did not have the same value in both files.

\section{$3 \quad$ Morphing}

This section describes the morphing algorithm that we designed and implemented.

Whereas the other models presented are predictive (cf. section 1.3), our model is an interpolated model based on morphing. The algorithm is presented in Fig. 3. It has three input parameters: the source and target files, containing the organ contour at the end of and at the beginning of the respiration cycle, respectively, and an integer which regulates the deformation, as explained below. There is one output parameter: the array of intermediate files generated at each step. 4DCT generates image files. Then, as explained in section 2.1, the Advantage4D software sorts the files. Next, the AdvantageSim software (by GE Medical systems) detects and stores the target organ contours.

As a first step, this algorithm reads the file corresponding to the initial image of the organ (at the beginning of expiration). Actually, the only information used is either each voxel is inside or outside the lung. So, the values that the algorithm deals with are ' 0 ' and ' 1 '. Actually, the contour of the organ is a set of voxels. When a contour voxel $V_{\text {source }}$ is found, each voxel located around takes the value that it has in the final image (at the end of expiration): ' 0 ' if it is outside the organ at the end, ' 1 ' otherwise. Fig. 4 illustrates the voxels that are taken into account around $V_{\text {source }}$ (the black voxel). This step is repeated until it obtains the final image of the organ 


\section{Program Morphing ;}

// Input and output parameters

Input: (Dicom_Struct) Source_File, Target_File ;

Input: (Number) Def_default ;

Output: (Dicom_Struct) Interm_Files[] ;

\section{// Main Program}

// Initialisations

iter $:=0$;

Intermediate_File[iter] := Source_File ;

// Main loop

While (Interm_File[iter] \& Target_File) Do

// Mean distance from the final organ contour D:=MeanDist (Interm_Files[iter], Target_File);

// Voxels to take into account around Vsource For each voxel Vsource of the contour Do

\author{
Dist := Def_default;

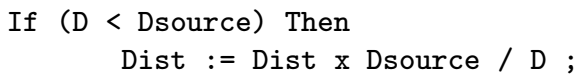 \\ End If ; \\ VoxelSet := VxTakenIntoAccount(Vsource,Dist) ; \\ // Voxel transformation \\ For each $\mathrm{v}$ in Voxelset Do \\ // $\mathrm{v}$ takes the value it has in the target file \\ Interm_Files[iter] (v) := Target_File(v) ; \\ End For ; // voxel transformation
}

End For ; // voxel around Vsource

// Next iteration

iter := iter+1 ;

Interm_File[iter] :=Interm_File[iter-1] ;
// Distance between Vsource and final Vsource Dsource := Distance(Vsource, Target_File) ;
End While ; // Main loop

End Program ;

Fig. 3: The morphing algorithm

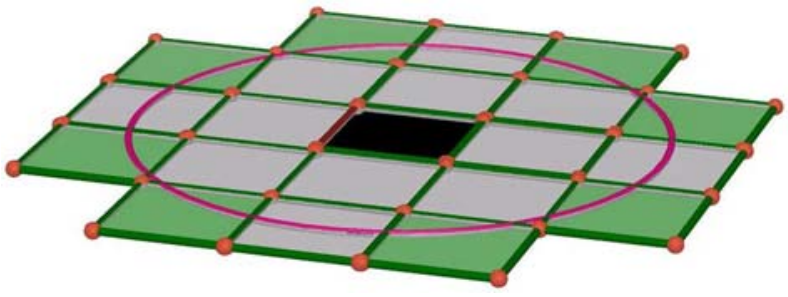

Fig. 4: Voxels that are taken into account at each step of the transformation

slice: this treatment is applied to each voxel of the organ contour of the considered image. The voxels of the initial image progressively take the ' $0-1$ ' values of the voxels of the final image. The result of this loop is an intermediate file which is not the final file. Thus, this loop is repeated until the intermediate file obtained is exactly equal to the final file: the intermediate file obtained will be considered as the new initial file during the next step. The intermediate files obtained, placed one after another in chronological order, create a motion. Thus, our purpose is to make this motion be close to the real lung motions of the patients.

The very first version of this algorithm took into account only the grey-coloured voxel of Fig. 4 . In fact, the obtained contours were very jagged, whereas in reality the contours are more circular. For this reason, the algorithm now takes into account grey-coloured and green-coloured voxels around each $V_{\text {source. }}$.
This algorithm implements a feature-based method (cf. section 1.1). Indeed, it is based on organ contour detection and its geometrical transformation. There is a kinetic regulation to determine the number of voxels taken into account around $V_{\text {source }}$. This method allows us to apply the transformation to different distances of $V_{\text {source }}$ : the voxels just in touch (8 voxels), or the voxels that are 2 voxels away from $V_{\text {source }}$ or $n$ voxels away from $V_{\text {source }}$. More precisely, the mean distance $D_{\text {source }}$ between $V_{\text {source }}$ and the voxels of the organ contour of the target image is calculated (by superimposing the two files). $V_{\text {source }}$ is not unique: the same mean distance is calculated for all the voxels of the contour. The algorithm obtains $D$, which is the mean distance of the mean distances. Each calculated $D_{\text {source }}$ is compared to $D$. If $D_{\text {source }}$ is less than $D$, the number of voxels transformed is the number defined as the input parameter; otherwise this input parameter is weighted by $D_{\text {source }} / D$.

Thus, the deformation applied is more important if $V_{\text {source }}$ is far from the final position of the organ contours. Furthermore, since these distances vary at each step of the algorithm, the deformation is faster during the construction of the first intermediate files than during the last files. Nevertheless, even if this version is very simple (2 grey levels and 2 dimensions), we wanted to validate our approach before improving it (multiple grey levels, 4 dimensions).

In order to validate our approach on a qualitative level, we compared the iterative organ contours obtained by morphing to the intermediate organ con- 
tours drawn by Advantage4D. We applied this technique to a patient and to different lung slices. In the next part of this paper, we present and analyze the results obtained.

\section{Results}

We selected four lung slices. We analyzed the scanned images from 4DCT and compared them to the images obtained by morphing. The first part of this section presents the results, the second part is a discussion regarding the advantages of morphing in radiotherapy, and the limitations of this algorithm and technique are presented in the last part.

\subsection{Morphing analysis}

Figs. 5 and 6 display the results obtained by $4 \mathrm{DCT}$ and morphing. The results are superimposed in Fig. 5: black lines represent the organ contours according to $4 \mathrm{DCT}$ and white lines represent the organ shapes according to the morphing algorithm. For slice 75 of the left lung, there were five 4DCT scans. We applied morphing as if the first and last scans (white shapes) were the lung image at maximal inspiration and maximal expiration, respectively. In that case, there are less than $2 \%$ different voxels at each step (each slice is composed of 512 by 256 voxels). As shown in Fig. 5, similar results are obtained with slice 0 . The most important difference is obtained with the right lung slice $-100: 2 \%$ different voxels between morphing intermediate images and 4DCT scans for one respiratory moment (34\%).

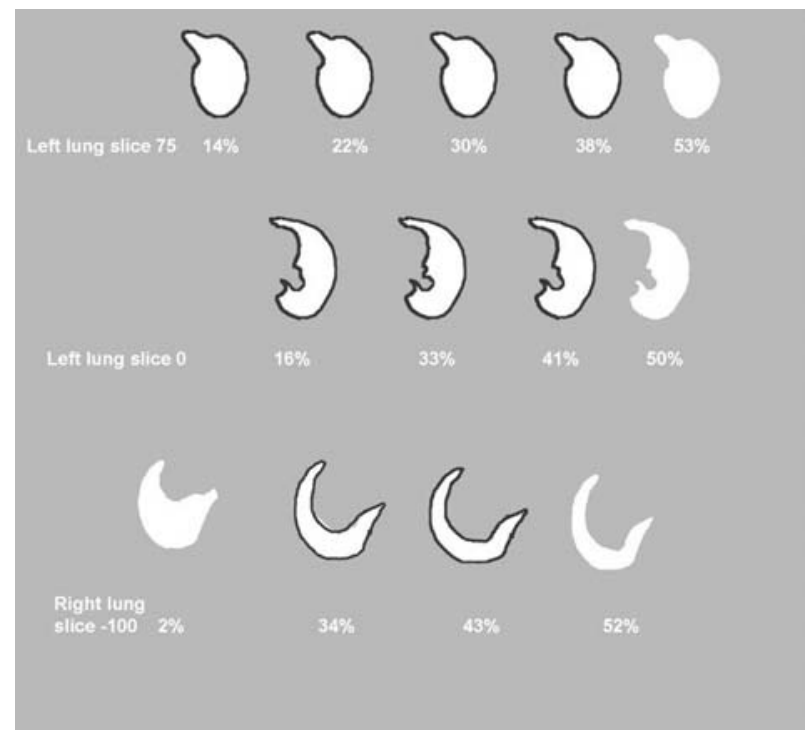

Fig. 5: Superimposing of the images obtained with morphing (white shapes) and 4DCT (black lines). Percentages are the accurate moments of each phase (Table 1)

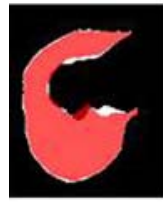

a)

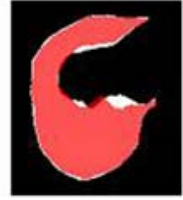

b)

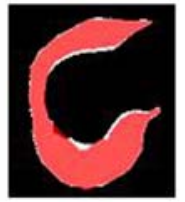

c)
Fig. 6: Right lung images superimposed, 4DCT in white, morphing in red, phase $10 \%$ (a), phase $20 \%$ (b), phase $40 \%$ (c), slice $-37.5 \mathrm{~mm}$

In Fig. 6, the lung obtained by morphing (in red) is superimposed over the lung image obtained with $4 \mathrm{DCT}$.

The differences between the images are acceptable. Indeed, these results tend to prove that the morphing algorithm that we have designed both describes and predicts lung motion. Now we have to account for the rhythm of the motion in morphing. Lung motion is relatively simple, but this may not be the case for other organs.

\subsection{Benefits of morphing}

Even if this version of our morphing implementation requires improvement, the present study suggests that applying morphing technique in radiotherapy is promising, and that it presents several considerable advantages.

The most important benefit is that it protects the patient from radiation. Indeed, the combination of 4DCT and morphing requires only two scans for each organ slice instead of six or more scans for each organ slice with the use of 4DCT alone. Morphing will not totally replace $4 \mathrm{DCT}$ scans, but it may considerably decrease the number of required scans.

The second advantage is that it improves contour detection and description. Indeed, morphing will enable the creation of an entire organ image at a precise moment $t$, even if there is no scan at $t$. Furthermore, morphing reduces the number of artifacts, since artifacts appear when no scan is available at $t$ for a specific slice. Therefore, morphing may be used in order to decrease the number of artifacts: considering lung slice -100 of Table 1 for example, instead of using a $2 \%$ scan in $10 \%$ phase, morphing could extrapolate a scan for $10 \%$ phase from the $2 \%$ scan and one other scan.

Finally, the description of the entire range of motion for an organ will be more precise. This will considerably benefit synchronization during the treatment phase.

\section{Conclusions}

These preliminary results concerning the use of morphing for organ deformation analysis are promising. 
The maximum morphing deviation is only $2 \%$. The lack of 4DCT scan patient data was highlighted. To overcome this limitation, morphing is a possible interpolation method. It can give intermediate organ contours in order to supplement 4DCT scan sorting. This will reduce the internal margin for the target volume and will enable the patient to be treated in gated-radiotherapy [16]. It will then be required to apply morphing in $3 \mathrm{D}$.

Our goal is to further refine the algorithm. Step by step, we will study ways to distinguish transformation speediness from organ wall elasticity and secondly to simulate the movement of the entire target organ. A further step will involve representing deformations of other organs and also taking into account the motion of organs at risk. Indeed, some organs may not have linear moves; there are accelerations and slowdowns. In addition, organ walls are not uniformly elastic. In order to have a global vision, it is necessary to apply separate speeds and distortions to simulate the motions of a group of organs.

\section{Acknowledgement}

The authors acknowledge financial support from LCC (Ligue Contre le Cancer), STIC (Soutien Technique aux Innovations Coûteuses) gating, région Franche-Comté, Cancéropôle Grand-Est, CAPM and Dr. R. Hamlaoui (CHU BesanCon) for organ delineations. We would like to thank Dr. G. Hruby for his help with translation and proof reading.

\section{References}

[1] Andrä, W., Danan, H., Eitner, K.: A novel magnetic method for examination of bowel mobility. Med. Phys. 2005, 32, 2942-2944.

[2] Atoui, H., Miguet, S., Sarrut, D.: A fast morphing-based interpolation for medical images: application to conformal radiotherapy. Image Anal Stereol, 2006, 25, 95-103.

[3] Bergeron, B. P., Sato, L., Rouse, R. L.: Morphing as a means of generating variation in visual medical teaching materials. Comput. Biol. Med., 1994, 24(1), 11-8.

[4] Berson, A. M., Emery, R., Rodriguez, L., Richards, G. M., Ng, T., Sanghavi, S., Barsa, J.: Clinical experience using gated radiation therapy: comparison of free-breathing and breathhold techniques. Int. J. Radiation Oncology Biol. Phys., 2004, 60, 419-426.

[5] Boldea, V., Sarrut, D., Clippe, S.: Lung deformation estimation with non-rigid registration for radiotherapy treatment. MICCAI 2003. Montreal (Canada), 2878, 770-777.
[6] Boldea, V., Sarrut, D., Sharp, G. C., Jiang, S. H., Choi, N. C.: Study of motion in a $4 \mathrm{D}$ CT using deformable registration, Int. J. Radiation Oncology Biol. Phys., 2005, 63, 499-500.

[7] Bro-Nielsen, M., Gramkow, G.: Fast fluid registration of medical images. Fourth International Conference on Visualisation in Biomedical Computing, VBC'96, Hamburg, Germany, 199, 267-276.

[8] Christensen, G. E.: Bayesian framework for image registration using eigenfunction. A. W. Toga (Ed.) Brian Warping, Academic Press, 1999, 5, 85-100.

[9] Collins, L., Evans, A. C.: Animal: Automatic nonlinear image matching and anatomical labeling. Toga, A. W. (Ed.) Brain Warping, Academic Press 1999, 8, 133-142.

[10] Davatzikos, G.: Spatial transformation and registration of brain images using elastically deformable models. Computer Vision and Image Understanding, Special Issue on Medical 1997, 66/2, 207-222.

[11] Davies, S. C., Hill, A. L., Holmes, R. B. et al.: Ultrasound quantification of respiratory organ motion in the upper abdomen, Br. J. Radiol, 1994, 67, 1096-1 102.

[12] Didier, A. L., Villard, P. F., Bayle, J. Y., Beuve, M., Shariat, B.: Breathing Thorax Simulation based on Pleura Physiology and Rib Kinematics. Information Visualisation MedVis, IEEE Ed., Zurich, Switzerland, 2007, 35-40.

[13] Heath, E., Seuntjens, J.: A direct voxel tracking method for four-dimensional Monte Carlo dose calculations in deforming anatomy. Medical Physics, 2006, 33/2, 434-445.

[14] ICRU report 62: International Commission on Radiation Units and Measurements. Prescribing, recording and reporting photon beam therapy, Supplement to ICRU Report 50, 1999.

[15] ICRU report 50. International Commission on Radiation Units and Measurements. Prescribing, recording and reporting photon beam therapy, 1993.

[16] Kubo, H. D., Len, P. M., Minohara, S., Mostafavi, H.: Breathing-synchronized radiotherapy program at the University of California Davis Cancer Center. Med. Phys., 2000, 27, 346-353. 
[17] Lester, H., Arridge, S. R.: A survey of hierarchical non-linear medical image registration, Pattern Recognition, 1999, 32, 129-149.

[18] Low, A. D., Nystrom, M., Kalinin, E., et al.: A method for the reconstruction of fourdimensional synchronized CT scans acquired during free breathing. Med. Phys., 2003, 30, 1254-1263.

[19] Mah, D., Hanley, J., Rosenzweig, K. E., et al.: Technical aspects of the deep inspiration breath-hold technique in the treatment of thoracic cancer. Int. J. Radiation Oncology Biol. Phys., 2000, 48, 1175-1185.

[20] Maintz, J. B. A., Viergever, M. A.: A survey of medical image registration. Medical Image Analysis, 1998, 2/1, 1-36.

[21] Montagner, J., Barra, V., Boire, J. Y.: A geometrical approach of multiresolution management in the fusion of digital images. 1st IEEE Visual Information Expert Workshop, Paris, France, 2006

[22] Moringlane, J. R.: Coordonnées polaires pour le système stéréotaxique de Talairach. Neurochirurgie, 1986, 32, 452-454.

[23] Musse, O., Heitz, F., Armspach, J. P.: Fast Deformable matching of 3D images over multiscale nested subspaces. Application to atlasbased MRI segmentation. Pattern Recognition, 2003, 36/8, 1881-1899.

[24] Nehmeh, S. A., Erdi, Y. E.: Four dimensional (4D) PET/CT imaging of the thorax, Med. Phys., 2004, 31, 3179-3186.

[25] Penska, K., Folio, L., Bunger, R.: Medical Applications of Digital Image Morphing. Journal of Digital Imaging, 2007, 20(3), 279-283.

[26] Rangarajan, A., Chui, H., Duncan, J. S.: Rigid point feature registration using mutual information. Medical Image Analysis, 1999, 3/4, 425-440.

[27] Rietzel, E., Pan, T., Chen, G. T. Y.: Fourdimensional computed tomography: Image formation and clinical protocol. Med. Phys., 2005, 32, 874-889.

[28] Rizzo, G., Scifo, P., Gilardi, M. C., Bettinardi, V., Grassi, F., Cerutti, S., Fazio, F.: Matching a computerized brain atlas to multimodal medical images. Neuroimage, 1996, 6, 59-69.
[29] Salomon, M., Heitz, F., Perrin, G. R., Armspach, J. P.: A massively parallel approach to deformable matching of 3D medical images via stochastic differential equations. Parallel Computing, Elsevier 2005, 31, 45-71.

[30] Santhanam, A. P., Willoughby, T., Shah, A., Meeks, S., Rolland, J. P., Kupelian, P.: Realtime simulation of $4 \mathrm{D}$ lung tumor radiotherapy using a breathing model. Proceedings of the 11th International conference on medical image computing and computer-assisted intervention 2008, 11 (pt 2), 710-717.

[31] Sarrut, D., Delhay, B., Villard, P. F., Boldea, V., Beuve, M., Clarysse, P.: A comparison framework for breathing motion estimation methods from 4D imaging. IEEE Transaction on Medical Imaging. 2007, 26(12), 1636-1648.

[32] Schweikard, A., Berlinger, K., Roth, M., Sauer, O., Vences, L.: Volumetric deformation model for motion compensation in radiotherapy. Medical Image Computing and ComputerAssisted Intervention MICCAI 2004, Saint Malo, France, 2004, 925-932.

[33] Sixel, K. E., Ruschin, M., Tirona, R., et al.: Digital fluoroscopy to quantify lung tumor motion: potential for patient-specific planning target volumes, Int. J. Radiation Oncology Biol. Phys., 2003, 57, 717-723.

[34] Stindel, E.: Bone morphing - 3D morphological data for total knee arthroplasty. Annual conference of The British Society for Computer Aided Orthopaedic Surgery, London, UK, 2006.

[35] Subsol, G., Thirion, J. P., Ayache, N.: Construction automatique d'atlas anatomiques morphéométriques à partir d'images médicales tridimensionnelles: application à un atlas du crâne. Medical Image Analysis, 1996, 2/1, 1-36.

[36] Talairach, J., Tournoux, P.: Co-planar stereotaxic atlas of the human brain. 3-dimension proportional system: an approach to cerebral imaging. Thieme Verlag, 1988.

[37] Thompson, P. M., Toga, A. W.: Wrapping strategies for intersubject registration. I. N. Bankman (Ed.), Handbook for Medical Imaging, Processing and Analysis, Academic Press, 2000, 36, 569-601.

[38] Thirion, J. P.: Diffusing models and applications. Toga, A. W., (Ed.) Brain wrapping, Academic Press 1999, 9, 143-155. 
[39] Thompson, P. M., MacDonald, D., Mega, M. S., Holmes, C. J., Evans, A. C., Toga, A. W.: Detection and mapping of abnormal brain structure with probabilistic atlas of cortical surfaces. Journal of Computed Assisted Tomography, 1997, 21/4, 567-581.

[40] Vérard, L., Allain, P., Travère, J. M., Baron, J. C., Bloyet, D.: Fully automatic identification of AC and PC landmarks on brain MRI using scene analysis. IEEE Transactions on Medical Imaging, 1997, 16/5, 610-616.

[41] Villard, P. F., Beuve, M., Shariat, B., Baudet, V., Jaillet, F.: Simulation of lung behaviour with finite elements: influence of biomechanical parameters. IEEE Conference on information visualization, London (GB), 2005, 9-14.

[42] Villard, P. F., Beuve, M., Shariat, B., Baudet, V., Jaillet, F.: Lung mesh generation to simulate breathing motion with a finite element method. IEEE Conference on information visualization, London (GB), 2004, 194-199.

[43] Weiss, P. H., Baker, J. M., Potchen, E. J.: Assessment of hepatic respiratory excursion, $J$. Nucl. Med., 1972, 13, 758-759.

[44] Wolthaus, J. W., Schneider, C., Sonke, J. J., Van Herk, M., Belderbos, J. S. A., Rossi, M. M. G., Lebesque, J. V., Damen, E. M. F.: Midventilation $\mathrm{CT}$ scan construction from fourdimensional respiration-correlated CT scans for radiotherapy planning of lung cancer patients. Int. J. Radiation Oncology Biol. Phys., 2006, 65(5), 1 560-1571.

[45] Wolthaus, J. W., Sonke, J. J., Van Herk, M., Damen, E. M.: Reconstruction of a timeaveraged midposition CT scan for radiotherapy planning of lung cancer patients using deformable registration. Med. Phys., 2008, 35(9), 3 998-4011.

[46] Yang, D., Lu, W., Low, D. A., Deasy, J. O., Hope, A. J., Naqa, I. E.: 4D-CT motion estimation using deformable image registration and $5 \mathrm{D}$ respiratory motion modeling. Med. Phys., 2008, 35(10), 4577-4590.

[47] Zanetti, E. M., Crupi, V., Bignardi, C., Calderale, P. M.: Radiograph-based femur morphing method. Med. Biol. Eng. Comput., 2005, 43(2), 181-8.

[48] Zhang, T., Keller, H., O'Brien, M. J., Mackie, T. R., Paliwal, B.: Application of the spirometer in respiratory gated radiotherapy. Med. Phys., 2003, 30, 3 165-3171.

Dr. Robert Laurent, Julien Henriet, Régine Gschwind,

Prof. Ing. Libor Makovicka, DrSc.

E-mail: Julien.henriet@pu-pm.univ-fcomte.fr IRMA/ENISYS/FEMTO-ST, UMR 6174 CNRS

Montbéliard, France 